\title{
Study of the Failure Mechanism of the Cu/epoxy Interface after Modifications by Means of Surface Chemical Reactions
}

\author{
Sam Siau ${ }^{\mathrm{a}}$ and Alfons Vervaet ${ }^{\mathrm{b}}$ \\ ${ }^{a}$ OCAS N.V., John Kennedylaan 3B-9060 Zelzate, Belgium, \\ ${ }^{\mathrm{b}}$ Department of ELIS, Universiteit Gent, Sint-Pietersnieuwstraat 41, 9000 Gent.
}

The chemical part of adhesion is studied at epoxy-copper interfaces. This is realized by creating epoxy surfaces with constant surface roughness by means of wet chemical pretreatments (sweller and oxidizer). These roughened epoxy surfaces are chemically transformed by nucleofilic substitutions using an anchoring molecule, 2-mercaptopyrimidine and glycerol. $\mathrm{Cu}$ is deposited by an electroless and electroplating scheme, followed by patterning. After patterning peel strengths are measured. The peeled epoxy surface and the backsides of the peeled Cu-strips are examined by means of XPS. These experiments are used to determine the influence of the interface atomic composition on the locus of failure during peeling.

\section{Introduction}

In build-up technology used in microelectronics the adhesion between epoxies and copper is essential. Sufficient adhesion can be realized by means of chemical and physical interactions and is often realized with a combination of sweller and oxidizer treatments $(1,2,3,4)$. The importance of the physical part of adhesion was demonstrated in (5). A relation was found between this part of adhesion and the roughness (maximal 3 $\mu \mathrm{m})$. The roughness is measured with the atomic force microscope (5) and is associated with a fractal structure developing as a consequence of the wet-chemical treatments. The AFM measured line length increase is proportional to the peel strength for limited oxidation treatment times. The chemical part of adhesion was practically constant in these experiments due to the fact that the number and types of chemical functions at the surface didn't vary much with an increase in oxidation treatment time (5).

Low surface roughness and a good epoxy-copper adhesion are requirements for optical and high frequency applications in build-up technology. This limits usage of increased roughness as adhesion improving treatments. The mechanical part of adhesion is heavily influenced by mechanical interlocking and will hence be influenced by the distribution, type, size, orientation, etc. of cavities formed near the interface, that will determine the degree of intertwining between both materials. As explored in previous research, the polymer composition, as well as the entire processing sequence used to process the sample, will influence cavity formation. The goal of this research is to investigate how the chemical composition of the surface influences the locus the failure during peeling. The chemical composition of the surface will hence influence adhesion of epoxy-copper joints. The hydroxyl functionalities present at the surface of the epoxy polymer can be substituted using surface reaction synthesis with several types of molecules with different types of properties (6). In this paper the change in surface chemical structure was realized with an anchor molecule (trichlorotriazine) and some heterocyclic $\mathrm{Cu}$-complexing organic molecules. After $\mathrm{Cu}$ electrodeposition peel strengths measurements were performed and the Cu-strips, as well as the epoxy layer were studied with XPS. It was 
shown that complexing agents had an important influence on the adhesion strength, which can be related to the interface composition. Apart of this contribution little literature is known on that subject.

In this present contribution 2-mercaptopyrimidine, a complexing agent, and glycerol, a source of different alcohol functions, are studied. The strategy is using a catalytic agent in order to optimize the addition of the anchor molecule on the epoxy surface, followed by introducing 2-mercaptopyrimidine and glycerol by nucleophilic substitution. $\mathrm{Cu}$ is deposited by electroless and electroplating and after patterning peel strengths are measured. The peeled epoxy and the Cu-strips are examined by means of XPS.

\section{Experimental}

In this part the methodology, based on (5), is described in order to obtain surfaces with minimal differences in roughness, but with a different chemical surface composition. The different surface compositions are obtained using chemical surface transformations with glycerol and 2-mercaptopyrimidine.

On top of an FR4 substrate 1-3 cm; thickness $1.2 \mathrm{~mm}$ a build-up layer of $40 \mu \mathrm{m}$ thickness was coated with the liquid Probelec 81/7081 photoimagable dielectric; provided by Vantico, an epoxy-fenol-novolac resin. This coated layer was flash dried for $15 \mathrm{~s}$ at $40^{\circ} \mathrm{C}$ and then convection dried for $30 \mathrm{~s}$ at $90^{\circ} \mathrm{C}$. After drying the layer was photoimaged and polymerized for $1 \mathrm{~h}$ at $150^{\circ} \mathrm{C}$. Hence a uniform polymer layer was obtained. This polymer layer was treated with different chemicals in order to change the properties of its surface: (i) Roughness formation: First, the sweller treatment, consists of a 7.5 min exposure to an aqueous solution containing 25-50 vol \% 2-(2-butoxyetoxy)ethanol (Shipley Circuposit Hole Prep 4125) at $80^{\circ} \mathrm{C}$ and second, the $\mathrm{KMnO} 4$ treatment for 10 min with an alkaline oxidizing solution exposure containing $55 \mathrm{~g} / \mathrm{L} \mathrm{KMnO} 4$ and 1.2 $\mathrm{mol} / \mathrm{L} \mathrm{NaOH}$ (Shipley Circuposit 3310) at $80^{\circ} \mathrm{C}$. (ii) First synthesis: Reaction of trichlorotriazine with the epoxy polymer): The surface of the epoxy polymer is treated with an anchoring component that allows synthesis of various groups. (iii) Second synthesis: Condensation reactions or more specific nucleofilic substitution reaction with the chemical anchors: Glycerine is bond with a condensation reaction and 2mercaptopyrimidine groups are covalently bond by nucleofilic substitution to the surface of the epoxy.(iv) Electrochemical deposition of copper and etching of peeling pattern: Copper is electrochemically deposited on the treated polymer layers using electroless $\mathrm{Cu}$ and electroplating of $\mathrm{Cu}$. A peeling pattern is etched in the copper layer that allows peel strength measurements (5). Hereafter more details are presented concerning:

(ii)First synthesis: Reaction of anchoring molecules with the epoxy polymer (Fig. 1).

The base component for adhering is trichlorotriazine (aka trichlorotricyanuryl) molecule. Reactions with and without a catalyst (glutidine) are realized.

(iii) Second synthesis: This is the reaction with the chemical groups on the anchors. After the first synthesis the samples are subjected to a second synthesis reaction using a nucleofilic substituting agent. These nucleofilically-substituted agents act as an adhesion promoter or complexion agent for the electrochemically deposited copper plated on top of the treated surface. Using this synthesis sequence the identity of the groups at the surface of the polymer can be changed. In Fig. 3 a and b synthesis reaction with glycerol and 2mercaptopyrimidine are depicted. The reaction with 2-mercaptopyrimidine was already partly studied is previous research (6). 

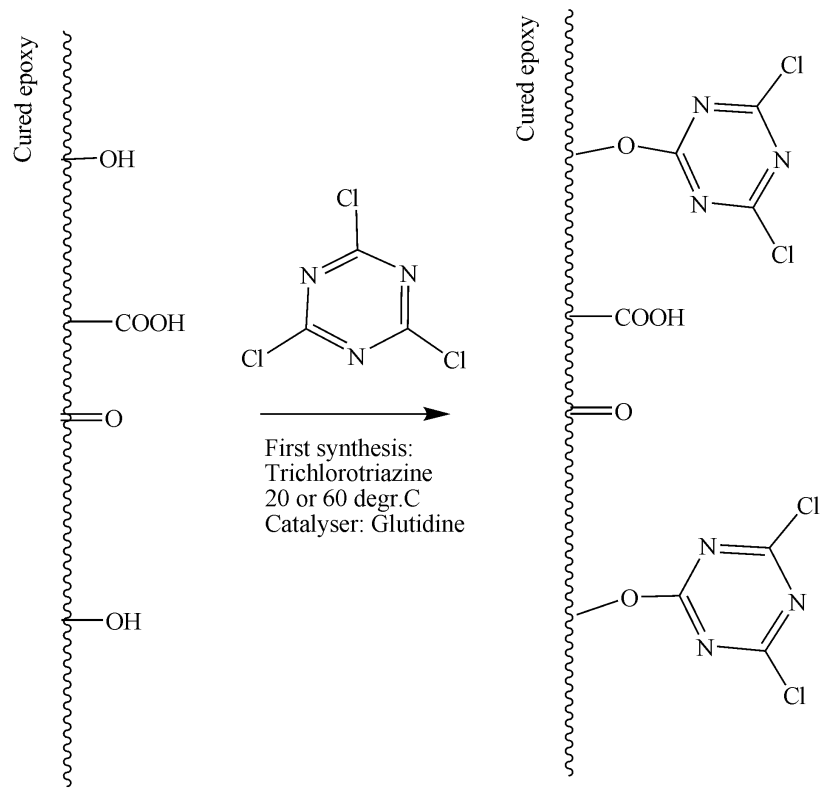

Figure 1. First synthesis reaction with trichlorotriazine either at 20 or $60^{\circ} \mathrm{C}$ and at the preoxidized surface.

With the combination of the first and second synthesis one active site on the surface can be replaced by at least two active groups. Figure 2 illustrates the change of the groups at the surface in a simplified manner. It is obvious that the treated surface, although it will have a roughness similar to the original surface, will have a different chemical composition that will affect the chemical part of adhesion.

Furthermore, the synthesis reaction only adds a limited thickness to the layer $(<1 \mathrm{~nm})$. The goal of this paper is to illustrate the importance of chemical adhesion due to certain groups at the surface in order to influence the adhesion strength. Furthermore just modifying the chemical groups at the surface and without modifying the mechanical interlocking the locus of failure can be shifted

(iv) Electrochemical deposition of copper and etching of peeling pattern. After the synthesis reactions, copper is electrochemically deposited onto the treated surfaces. This electrochemical deposition of copper consists of two depositions. The first stage is an electroless copper-plating step Shipley process (1), followed by the electroplating of $\mathrm{Cu}$ as a second stage. This way a $15 \mu \mathrm{m}$ thick copper layer was deposited on top of the modified polymer surfaces. After each $\mathrm{Cu}$ deposition the samples were annealed in a convection oven at $150^{\circ} \mathrm{C}$ for $1 \mathrm{~h}$. After the deposition of copper, a pattern of $3 \mathrm{~mm}$ strips is etched in the layer using an acid $\mathrm{CuCl}_{2} / \mathrm{HCl}$ etching liquid (1), and these strips were peeled from the polymer surface. This peel test was performed using a Dage Series 4000 with a TP $5 \mathrm{~kg}$ cartridge. Further details about the peel strength measuring mechanism and the used plating can be found in previous publications $(1,5)$. 

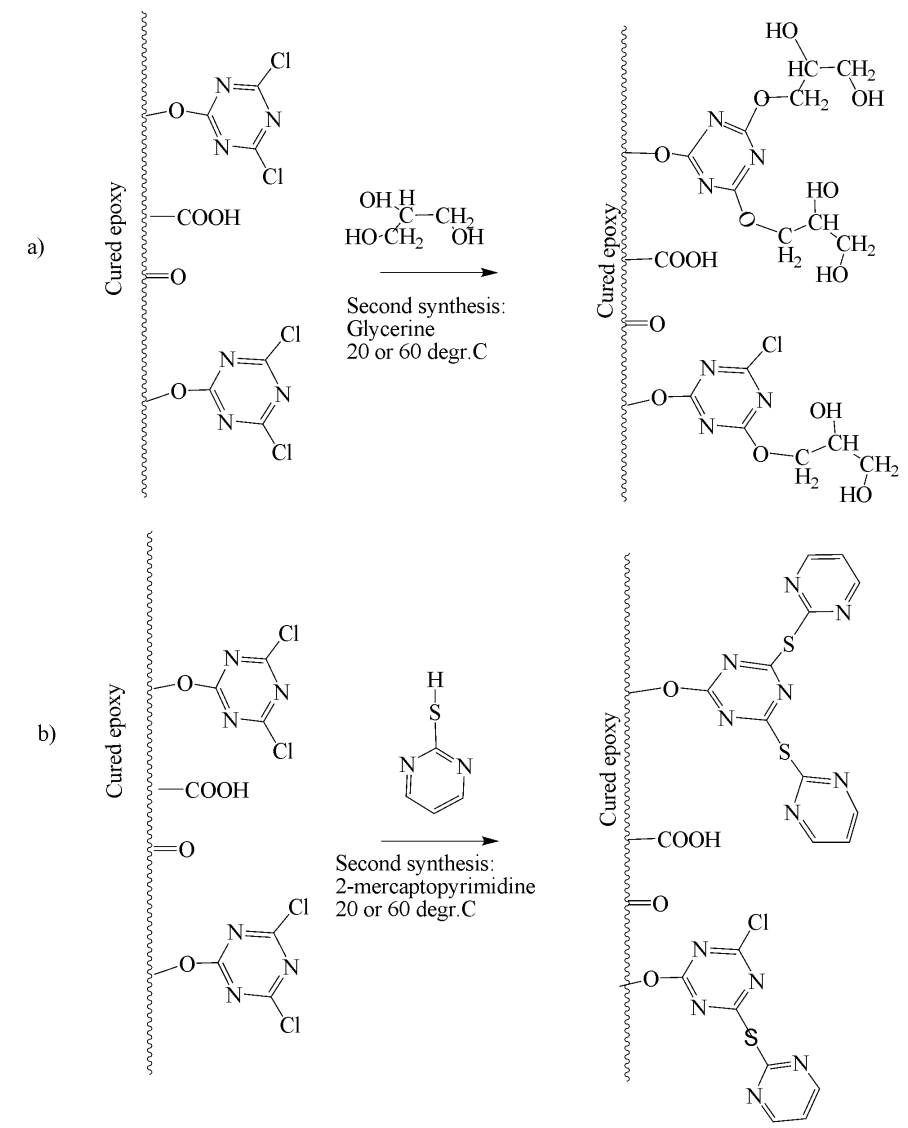

Figure 2. Second synthesis reaction (either 20 or 60 degr. C) on the preoxydised polymer surface: a) with glycerol, b) with 2-mercaptopyrimidine.

\section{Results}

XPS measurements of the peeled epoxy polymer surface and the copper strip are presented in Table 1. Experiments were realized in 2 points for the $\mathrm{Cu}$-strip in order to study the homogeneity of the peeled surfaces transformed with 2-mercaptopyrimidine and glycerol. The compositions measured in Tabel 1 show that for both synthesized surfaces the copper and polymer layer are enough intertwined to allow copper to be present near the outermost surface of the print and for Pd and Sn (resulting from the electroless activation step) to be present on the back sides of the copper strip. This indicates that the failure of the epoxy-copper joint during peeling is near the interface between both materials. However, some differences are measured between the various samples.

The peel forces for the treated surfaces with glycerol and 2-mercaptopyrimidine are shown in Tabel 2. 
TABEL 1: XPS chemical composition (in atomic\%) of the prints and the $\mathrm{Cu}$-strips from the interfaces of the $\mathrm{Cu} /$ epoxy interface with different surface group transformations: a) glycerol, b) 2mercaptopyrimidine

a) Glycerol

\begin{tabular}{|c|c|c|c|c|c|l|}
\hline $\mathbf{O}$ & $\mathbf{C}$ & $\mathbf{N}$ & $\mathbf{P d}$ & $\mathbf{S n}$ & $\mathbf{C u}$ & \\
\hline 12 & 71.06 & 1.09 & 0.59 & 0.15 & 15.11 & print \\
\hline 24.11 & 26.53 & 0.45 & 0.17 & 0.06 & 47.69 & Cu-strip \\
\hline 12.19 & 48.99 & 0.8 & 1.19 & 0.42 & 36.42 & Cu-strip \\
\hline
\end{tabular}

b) 2-Mercaptopyrimidine

\begin{tabular}{|c|c|c|c|c|c|l|}
\hline $\mathbf{O}$ & $\mathbf{C}$ & $\mathbf{N}$ & $\mathbf{P d}$ & $\mathbf{S n}$ & $\mathbf{C u}$ & \\
\hline 8.65 & 88.17 & 0.83 & & 0.01 & 2.33 & print \\
\hline 15.33 & 33.75 & 1.08 & 0.62 & 0.16 & 49.05 & Cu-strip \\
\hline 9.98 & 62.64 & 0.78 & 0.32 & 0.11 & 26.16 & Cu-strip \\
\hline
\end{tabular}

TABEL 2. Peel forces with the estimated experimental error for the $\mathrm{Cu} /$ epoxy interfaces formed with glycerol and 2-mercaptopyrimidine

\begin{tabular}{|c|c|c|}
\hline & Force in N for 3mm strip & Estimation of sigma \\
\hline Glycerol & 0.465 & 0.169 \\
\hline 2-Mercaptopyrimidine & 1.482 & 0.696 \\
\hline
\end{tabular}

The deposition of $\mathrm{Cu}$ was very flat for gycerol and showed a bubbled surface structure for 2-mercaptopyrimidine. The thickness obtained was $15 \mathrm{um}$. The thermal decomposition of 2-mercaptopyrimidine with formation of sulfites can probably be associated to this observation.

\section{Discussion}

The goal of this work is to study the failure mechanism of the interface by determining the locus of failure and evolution of the peel force for the interfaces realised with gycerol and 2-mercaptopyrimidine. In earlier work (7) the following correlations were obtained: i) the $\mathrm{N} / \mathrm{Cu}$ ratio and the ability of complexation, ii) a high carbon content of the copper strip and a high peel strength, iii) low peel strength and high oxygen content on the back side of the peeled copper strip.

Based on these observations a protocol is deduced in this paper in order to evaluate the locus of failure and the peel force for the new interfaces. The following protocol is proposed: 
1. N/Cu values are a measure for possible complexation effects. The highest values are found in the interface because the synthesis took place near the interface between both materials.

2. Pd and Sn percentages can be used to determine the small shifts in location of failure (epoxy, interface or $\mathrm{Cu}$ ). This is possible because these elements were the catalyst for the deposition of the electroless copper on top of the modified polymer surfaces.

3. Low C- and high O-values of the Cu-strips can be a measure for low peel forces due to excessive sublayer damage in the upper parts of the polymer layer.

4. High C-values on the backside of the peeled copper strips can be are related to failure in the epoxy. Usually high peel strengths are achieved.

5. Low Cu-content in the print is related to failure in the epoxy.

The reversed statements are also true.

The aim of this contribution is to study the failure mechanism of the interface. This will be realised by testing the proposed protocol, which is based on previous results with iminodiacetic acid and imidazole (7), with the XPS chemical compositions of the interfaces formed with glycerol and 2-mercaptopyrimidine (Table 1). Table 3 is presented so that in each column or combination of them one element of the protocol is shown. (first item N/Cu; second item Pd, Sn; third and fourth item $\mathrm{O}, \mathrm{C}$; fifth item $\mathrm{Cu}$ ) The evolution of the values in these columns permits to deduce qualitatively the locus of failure for the new interfaces and to obtain an estimation of the peel force in order to verify the experiments in Tabel 2 for glycerol and 2-mercaptopyrimidine.

- Glycerol

By comparing the N/Cu values for all prints we see that the lowest value is obtained for glycerol. This can be understood since glycerol has no $\mathrm{N}$-atoms and the complexing doesn't occur with the $\mathrm{N}$-atoms of the underlying trichorotriazine due to the screening effect of glycerol. The presence of $\mathrm{N}$ is an indication that the failure occurs in the vicinity of the interface. In glycerol the complexing occurs with the O-atoms so that we can explain why the lowest value for the $\mathrm{N} / \mathrm{Cu}$ ratio (first item of protocol) are obtained. The place of the locus of failure in this interface is indicated by the Pd, Sn-values. Nearly the same values for the print and the Cu-strip are obtained so that we can conclude that failure is located on top of the added molecules (second item of the protocol). A lower Ccontent and a higher $\mathrm{O}$-content for the $\mathrm{Cu}$-strip compared to 2-mercaptopyrimidine confirm this result (third item of the protocol). These values are also an indication that the polymer is weakly attached to $\mathrm{Cu}$ (fourth item of the protocol). High copper content is related to failure in the copper rich region (fifth item of the protocol). Thus it can be concluded that the locus of failure is situated in the interface at the side of the copper layer and the peel force is low compared to 2-mercaptopyrimidine.

\section{- 2-Mercaptopyrimidine}

The low value for $\mathrm{N} / \mathrm{Cu}$ is an indication that failure takes place outside the interface (first item of the protocol). Where the failure is situated depends on the Pd, Sn-values in the print and the Cu-strip: the highest value is obtained for the Cu-strip and nearly immeasurable one for the print so that the failure is at the side of the epoxy (second item of the protocol). Higher $\mathrm{C}$ and lower $\mathrm{O}$ compared to glycerol indicate that the peel force 


\begin{tabular}{|c|c|c|c|c|c|c|}
\hline \multicolumn{7}{|c|}{$\begin{array}{l}\text { TABEL } 3 \text { The XPS-measurer } \\
\text { interfaces a) IDA pH=7, b) } \\
\text { Mercaptopyrimidine and e) Glyce } \\
\text { them represents one item of the } p \\
\text { * second place measured } \\
\star \star \text { not measurable }\end{array}$} \\
\hline & $\begin{array}{c}\text { N/Cu } \\
(\%)\end{array}$ & 0 & C & Pd & Sn & $\mathrm{Cu}$ \\
\hline & \multicolumn{6}{|c|}{ a) IDA $\mathrm{pH}=7$} \\
\hline print & 25.99 & 14.16 & 61.49 & 2.32 & 0.42 & 17.16 \\
\hline Cu-strip & 1.70 & 39.76 & 14.78 & 0.02 & 0.02 & 44.66 \\
\hline \multirow[t]{2}{*}{ Cu-strip } & 1.42 & 14.89 & 20.41 & 0.89 & 0.02 & 62.72 \\
\hline & \multicolumn{6}{|c|}{ b) IDA $\mathrm{pH}=10$} \\
\hline print & 65.13 & 2.94 & 93.73 & 0.10 & 0.02 & 1.95 \\
\hline Cu-strip & 9.79 & 9.5 & 59.41 & 0.66 & 0.38 & 27.37 \\
\hline Cu-strip & 13.99 & 8.06 & 72.43 & 0.45 & 0.32 & 16.44 \\
\hline print* & 2.11 & 12.96 & 52.00 & 0.12 & 0.03 & 34.17 \\
\hline Cu-strip* & 7.84 & 7.71 & 72.21 & 0.37 & 0.05 & 18.23 \\
\hline \multirow[t]{2}{*}{ Cu-strip* } & 0.94 & 24.11 & 26.53 & 0.17 & 0.06 & 47.69 \\
\hline & \multicolumn{6}{|c|}{ c) Imidazole } \\
\hline print & 199.69 & 6.29 & 74.41 & $\star *$ & 0.04 & 6.43 \\
\hline Cu-strip & 19.31 & 6.46 & 48.26 & 0.28 & 0.10 & 37.64 \\
\hline \multirow[t]{2}{*}{ Cu-strip } & 25.79 & 5.86 & 52.93 & 0.48 & 0.10 & 32.30 \\
\hline & \multicolumn{6}{|c|}{ d) 2-Mercaptopyrimidine } \\
\hline print & 35.62 & 8.65 & 88.17 & ** & 0.01 & 2.33 \\
\hline Cu-strip & 2.20 & 15.33 & 33.75 & 0.62 & 0.16 & 49.05 \\
\hline \multirow[t]{2}{*}{ Cu-strip } & 2.98 & 9.98 & 62.64 & 0.32 & 0.11 & 26.16 \\
\hline & \multicolumn{6}{|c|}{ e) Glycerol } \\
\hline print & 7.21 & 12 & 71.06 & 0.59 & 0.15 & 15.11 \\
\hline Cu-strip & 0.94 & 24.11 & 26.53 & 0.17 & 0.06 & 47.69 \\
\hline Cu-strip & 2.20 & 12.19 & 48.99 & 1.19 & 0.42 & 36.42 \\
\hline
\end{tabular}

will be higher than in the case of glycerol (third item of the protocol). Low Cu-content in the print is an indication that the failure takes place in the epoxy (fourth item of the protocol). The conclusion is that the locus of failure is situated in the epoxy and that the peel force is higher than in the case of glycerol.

The locus of failure cannot exactly be determined due to the limits of the experiments and the measurement techniques. In the experiments the roughness of the surface is responsible for the intertwining between both layers. Both synthesis reactions will alter the properties of the chemical groups. The thickness of the layer added by the synthesis reaction will be negligible $(<1 \mathrm{~nm})$. However, the penetration depth of the chemicals can be higher than only the upper atomic layers of the polymer. The photoelectrons of the XPS analysis have only a limited escape depth and therefore determine the composition of the upper atomic layers10 nm of material. The fact that the backsides of the strips and the remaining surface have different chemical properties according to XPS illustrates that the location of the failure of the epoxy-copper joint must be in the vicinity of the interface 
between both materials. The highest $\mathrm{N}$ contents should be registered near the upper regions of the epoxy polymer layer.

In the next part, based on the proposed protocol, the loci of failure are deduced for all the studied complexing agents. This is realised by resuming the results of Tabel 3 in Tabel 4 with the quotes,,,,$++++++++/$ - and - representing the selected limits between the brackets. The complexants of Tabel 4 are discussed in the light of the protocol: the first column indicates the evolution of $\mathrm{N} / \mathrm{Cu}$ in the print from - to +++ indicating the locus of failure: - in the epoxy or copperrich region to +++ nearest to the interface; the second column combined with the first, third and fourth column situates the failure in the interface + side epoxy or - side copper. All conclusions and general conclusions are also presented in Tabel 4.

TABEL 4 Resume of the results of Table 5 based on the quote,,,,$++++++++/-$ and - with the limits of the quotes in the brackets. The figures in bold represent the peel force. The conclusions are based on the protocol.

* measurement on a second place

\begin{tabular}{|c|c|c|c|c|c|}
\hline & $\begin{array}{l}\mathrm{N} / \mathrm{Cu} \\
\text { print }\end{array}$ & $\begin{array}{c}\text { Pd } \\
\text { print }\end{array}$ & $\begin{array}{c}\mathrm{C} \\
\mathrm{Cu}-\mathrm{strip}\end{array}$ & $\begin{array}{c}\mathrm{Cu} \\
\text { print }\end{array}$ & General Conclusion \\
\hline 1) IDA pH=7 & $\begin{array}{c}+ \\
(5-40)\end{array}$ & $\stackrel{+}{+}(>0.5)$ & $\begin{array}{c}+ \\
(<25) \\
\mathbf{1 , 4}\end{array}$ & $\begin{array}{c}++ \\
(10-20)\end{array}$ & \\
\hline Concusion & interface & $\begin{array}{l}\text { copperrich } \\
\text { side }\end{array}$ & $\begin{array}{l}\text { poor } \\
\text { epoxy }\end{array}$ & $\begin{array}{l}\text { copperrich } \\
\text { side }\end{array}$ & $\begin{array}{l}\text { Failure in copper rich region } \\
\text { based on column }\end{array}$ \\
\hline 2) $\mathrm{IDA} \mathrm{pH}=10$ & $\begin{array}{c}++ \\
(40-80)\end{array}$ & $\begin{array}{c}+/- \\
(0.1-.5)\end{array}$ & $\begin{array}{c}+++ \\
(>50) \\
2,0\end{array}$ & $\begin{array}{c}- \\
(<5)\end{array}$ & \\
\hline 2) Conclusion & interface & not defined & $\begin{array}{l}\text { much } \\
\text { epoxy }\end{array}$ & epoxy side & Failure interface side epoxy \\
\hline 3) IDA pH=10* & $\begin{array}{c}- \\
(<5)\end{array}$ & $+/-$ & $\begin{array}{c}++ \\
2.8 \\
\end{array}$ & $\begin{array}{c}+++ \\
(>20)\end{array}$ & \\
\hline 3) Conclusion & $\begin{array}{l}\text { outside } \\
\text { interface }\end{array}$ & not defined & in epoxy & in copper & $\begin{array}{l}\text { Failure not defined partial in } \\
\text { Cu and partial in epoxy }\end{array}$ \\
\hline 4) Imidazole & $\begin{array}{c}+++ \\
(>80)\end{array}$ & $(<0.1)$ & $\begin{array}{c}++ \\
(25-50) \\
\mathbf{1 . 5}\end{array}$ & $\begin{array}{c}+ \\
(5-10)\end{array}$ & \\
\hline 4) Conclusion & interface & side epoxy & in epoxy & low copper & failure interface side epoxy \\
\hline $\begin{array}{l}\text { 5) 2-Mercapto- } \\
\text { pyrimidine }\end{array}$ & + & $\mathrm{C}_{\mathrm{C}} \mathrm{J}$ & $\begin{array}{l}++ \\
1.5 \\
\end{array}$ & 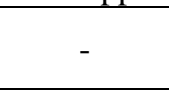 & \\
\hline 5) Conclusion & interface & side epoxy & in epoxy & $\begin{array}{l}\text { very low } \\
\text { copper }\end{array}$ & Failure epoxy \\
\hline 6) Glycerol & + & + & $\begin{array}{c}++ \\
0.47\end{array}$ & ++ & \\
\hline 6) Conclusion & interface & $\begin{array}{l}\text { copperrich } \\
\text { side }\end{array}$ & in epoxy & $\begin{array}{l}\text { copperrich } \\
\text { side }\end{array}$ & $\begin{array}{l}\text { Most chance fo failure } \\
\text { interface side } \mathrm{Cu}\end{array}$ \\
\hline
\end{tabular}


The results of Tabel 4 are presented in Fig 3. This figure presents the build-up layer starting with the epoxy-layer, the 2 chemical transformations, the seed layer and finally the copper layer. The dotted lines in this figure present the place of failure of the different agents 1) iminodiacetic acid $\mathrm{pH}=7$; 2) and 3) iminodiacetic acid $\mathrm{pH}=10$; 4) imidazole; 5) 2-mercaptopyrimidine; 6 glycerol. XPS measurements show that the chemical composition near the interface (top $10 \mathrm{~nm}$ of the polymer surface) has been altered by the procedure and permits us to locate the failure in the interface. Experimental variations (see 2), 3) in Tabel 4) and the conclusion in 3) show that the surfaces are not homogeneous.

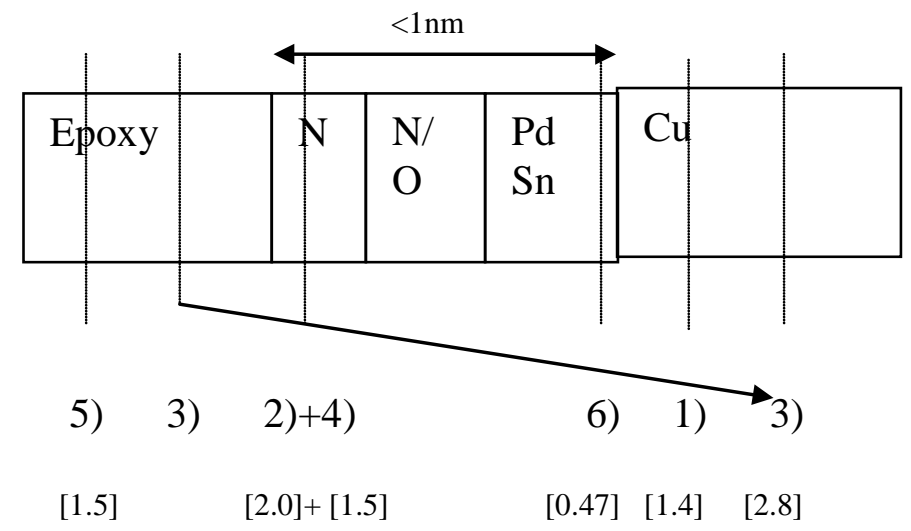

Figure 3 Representation of the build-up of the interface based on the chronology of the surface transformations and the loci of failure for 1) iminodiacetic acid $\mathrm{pH}=7 ; 2$ ) and 3) iminodiacetic acid $\mathrm{pH}=10$ (Failure not defined partial in $\mathrm{Cu}$ and partial in epoxy); 4) imidazole; 5) 2-mercaptopyrimidine; 6) glycerol. Underneath each locus the approximate peel force is shown in the brackets.

Peel force measurements (see the values in the bracket in Fig. 3, Table 2 and Table 4) confirm that the groups located at the surface of the polymer have a pronounced influence on the adhesion strength of electrochemically deposited copper. C in the Cu-strips (Tabel 4) is qualitatively correlated to the peel force. The high error on the peel strength, observed for 2-mercaptopyrimidine (see Tabel 2), can probably be attributed to the instability of $S$ in that molecule due to the oxidation of $S$ to sulfites. Some particularities of 2-mercaptopyrimidine were discussed in (6).

\section{Conclusion}

Though it is difficult to compare different chemical transformations of the surface and the obtained interfaces, the proposed protocol permitted with success to interpret XPSmeasurements, to deduce the failure mechanism and finally to obtain the loci of failure. At this moment the experiments are not optimized. The study of the evolution of these loci of failure can give indications concerning the strategies to follow in order to obtain 
desired peel forces $>1.5 \mathrm{~N} / \mathrm{mm}$ necessary for applications in microelectronics. Optimization of the result of iminodiacetic acid $\mathrm{pH}=10$ will be the scope of a next publication.

\section{References}

1. S. Siau, A. Vervaet, E. Schacht and A. Van Calster, J. Electrochem. Soc. 151, 2, C133 (2004)

2. S. Siau, A. Vervaet, A. Van Calster, I. Swennen and E. Schacht, J. Electrochem. Soc. 151, 8, J54 (2004)

3. S. Siau, A. Vervaet, S. Nalines, A. Van Calster and E. Schacht, J. Electrochem. Soc. 151, 12, C816 (2004)

4. S. Siau, A. Vervaet, S. Nalines, A. Van Calster and E. Schacht, J. Electrochem. Soc. 151, 12, C831 (2004)

5. S. Siau, A.Vervaet, L. Van Vaeck, E. Schacht, U. Demeter and A.Van Calster , Journal of the Electrochemical Society, 152, 6, C442-C455 (2005)

6. S. Siau, A. Vervaet, E. Schacht, S. Degrande, Kristof Callewaert and Andre Van Calster, Journal of the Electrochemical Society, 152, 9, D136-D150 (2005)

7. S. Siau, A.Vervaet, E. Schacht, U. Demeter and A. Van Calster, Thin Solid Films, 495, 348-356 (2006)

8. V.I. Mur Russ. Chem. Rev., 33, 92 (1964) 